\title{
Outflows and Accretion in Young Stellar Objects
}

\author{
Nuria Calvet \\ Smithsonian Astrophysical Observatory, 60 Garden St., MS 42, \\ Cambridge, MA 02138, USA
}

\begin{abstract}
Outflows in young stellar objects are powered by accretion, and $\sim 0.1$ of the accreted material is lost in the outflow. Observational evidence is analyzed in the context of models for the origin of the wind. Winds in FU Ori objects are clear examples of disk winds. In Classical $\mathrm{T}$ Tauri stars, there is evidence for the existence of a wide angle wind at scales $<100 \mathrm{AU}$, which supports the $\mathrm{X}$-wind model prediction that narrow jets are the result of density/temperature enhancement towards the axis of the system. However, recent HST observations of the DG Tau jet indicate that the opening angle of the wind is more confined than predicted by the X-wind model, in better agreement with disk wind theories.
\end{abstract}

\section{Introduction}

The presence of outflows in young stellar objects has been inferred from the $\mathrm{P}$ Cygni profiles of some of their emission lines. First attempts to interpret the properties of the wind of Classical T Tauri stars (CTTS) date back decades (Kuhi 1964); all of these early attempts considered the wind region to be spherical and stellar in origin (Hartmann et al. 1982, 1990; Natta, Giovanardi, \& Palla 1988; Natta \& Giovanardi 1990). In fact, with the advent of IUE and the first UV spectra of CTTS, an "amplified solar analogy" explanation was proposed to explain the excess energy above photospheric fluxes that characterizes these stars (Giampapa et al. 1980), and an MHD origin for the wind, similar to that applied to late type stars, was proposed (Hartmann et al. 1982).

A puzzling feature in those early stages of research was the presence of redshifted absorption components in some of the emission lines, indicating infall of matter. A whole subclass of objects was identified, the YY Orionis stars, in which these features Were observed (Walker 1972; Wolf et al. 1977). However, their existence as a separate class faded into oblivion as more and more cases were identified in which both blueshifted and redshifted absorption components appear in emission lines in the spectra of the same stars, and even in the same line. Nonetheless, the puzzle stayed because to have simultaneous outflow and infall could not be understood with a spherical emitting region.

Another disturbing fact for the stellar analogy was the overall energy budget in CTTS. In most stars, energy losses were of the order of $10 \%$ of the stellar luminosity, and in some stars the losses could be as high or even higher than 
the stellar luminosity itself (cf. Figure 6.3 in Hartmann 1998). This losses are much higher than those found in any star with activity powered by dynamos.

By the middle of the 1980's, the presence of accretion disks around CTTS was becoming accepted. They have been proposed as the source of the excess emission in these objects in the pioneering paper by Lynden-Bell \& Pringle (1974), but the hypothesis was not widely accepted until the first IRAS fluxes showed infrared excesses roughly consistent with the emission of accretion disk models (Rucinski 1984). Additionally, excess emission at millimeter wavelengths could be accounted for by disk emission (Beckwith et al. 1990), and disks were finally imaged by HST by the end of the century (Burrows et al. 1996).

About the same time, instabilities in the accretion disk around a CTTS, which raised the mass accretion rate by several orders of magnitude, were successfully proposed as the explanation for the FU Ori phenomenon (Hartmann \& Kenyon 1996, and references therein). These instabilities could naturally explain the two fold increase of the object luminosity, as well as the line doubling and wavelength-dependent spectral type and rotational broadening observed in the FU Ori objects.

The energy budget can be explained if CTTS are surrounded by accretion disks, because accretion energy could naturally account for the excess energy. In the next sections, I will discuss the present picture for the inner accretion disk region of CTTS. It is in this region where matter is loaded onto the star and from which the wind is launched.

\section{Magnetospheric accretion}

Even if accretion is powering most of the excess energy above photospheric levels in CTTS, the stars themselves are very active. Indications of the properties of the stellar photosphere come from study of the weak-line T Tauri stars, WTTS, which are in the same region of the HR diagram but lack accreting disks. These stars still have emission lines, and their UV and X-ray emission is comparable or higher than those of the most active late type stars (Costa et al. 2000).

Magnetic fields of the order of a few KG have being measured in CTTS with detailed modeling of the Zeeman broadening of magnetically sensitive absorption lines in the infrared (Johns-Krull et al. 1999b). In addition, circular polarization has been measured in the He I 5876 emission line (Johns-Krull et al. 1999a), indicating not only strengths of order of $\mathrm{KG}$, but also the existence of a large scale ordered field.

Magnetic fields of this strengths could prevent the disk to reach the star if the rate of mass accretion was low enough. We can get estimates of this rate from observations of the excess emission in CTTS relative to photospheric fluxes. If this emission is identified with accretion luminosity, $L_{c c}=G M_{*} \dot{M} / R_{*}$, with $M_{*}$ and $R_{*}$ the stellar mass and radius and $\dot{M}$ the mass accretion rate, then knowing the mass and radius from the position in the HR diagram, the mass accretion rate can be estimated. Measurements of the excess emission in the blue and ultraviolet results in an average mass accretion rate of $10^{-8} M_{\odot} y r^{-1}$ in CTTS (Gullbring et al. 1998).

For spherical accretion of matter around a star, accretion if halted when the magnetic pressure is equal to the ram pressure of the flow, $B^{2} \sim 4 \pi \rho v^{2}=\dot{M} v / r^{2}$, 
where $v$ is the free-fall velocity and $\rho$ the density of the flow. For a dipolar field, the truncation radius is

$$
r_{t}=7 R_{*}\left(\frac{B}{1 K G}\right)^{4 / 7}\left(\frac{\dot{M}}{10^{-8} M_{\odot} y r^{-1}}\right)^{-2 / 7}\left(\frac{M_{*}}{0.5 M_{\odot}}\right)^{-1 / 7}\left(\frac{R_{*}}{2 R_{\odot}}\right)^{5 / 7}
$$

where the normalization is done to typical quantities for CTTS. For disk accretion, the truncation radius is $R_{t} \sim \gamma r_{t}$, with $\gamma \sim 1 / 3-2 / 3$ (Ghosh \& Lamb 1979; Königl 1991; Shu el al. 1994), so for typical parameters the inner disks of CTTS will be truncated by the magnetic field at a few stellar radii. Disk matter reaches the star free-falling along the magnetic field lines, and merges with photospheric material through an accretion shock on the stellar surface. Ample evidence supports this magnetospheric model of accretion.

The profiles of the emission lines in typical CTTS could not be explained by wind profiles. They showed blue-shifted absorption components, but superimposed on emission lines with peaks that nearly centered at zero velocity or even slightly blueshifted (Edwards et al. 1994). Moreover, the already mentioned red-shifted absorption components were often present (Edwards et al. 1994), so they could not be properly classified in the P Cygni category. Many attempts to model the profiles with stellar (Hartmann et al. 1990) or boundary-layer (Calvet et al. 1992) winds were not successful. On the other hand, magnetospheric models can beautifully reproduce observed line profiles (Hartmann et al. 1994; Muzerolle et al. 1998a, b, 2001), and the red-shifted absorption arises naturally if the line of sight intercepts flow falling in front of the hot accretion shock.

Another prediction of the magnetospheric model of accretion is that a shock forms at the stellar surface. On this shock, potential energy of the infalling matter, which amounts to essentially the accretion luminosity for typical values of $R_{t}$, thermalizes, and emerges as excess flux. Predictions for the accretion shock emission compare very well with the overall spectral energy distribution of the excess flux in CTTS, specially in the blue and ultraviolet (Calvet \& Gullbring 1998; Gullbring et al. 2001).

Therefore, it is well accepted now that the inner disks of CTTS are truncated at a few stellar radii, the broad emission lines are formed in the magnetospheric flow, and the excess emission is due to the accretion shock. The validity of this model has now been proved for a large range of masses in pre-main sequence late-type stars, from brown dwarfs (Muzerolle et al. 2003a) to intermediate mass TTS (Calvet el al. 2003). A similar model seems to account for the emission properties of the Herbig Ae stars (Muzerolle et al. 2003b).

\section{Origin of the wind}

While the overall picture of magnetospheric accretion is well accepted, it is less clear where the wind originates in CTTS. Mass ejection certainly exist. The blue-shifted absorption components with up to several hundred $\mathrm{km} \mathrm{s}^{-1}$ shifts cannot be explained otherwise. Moreover, strong highly collimated jets emitting in hydrogen and forbidden lines are seen emerging from the most active among the CTTS, and at large scales, effects of these winds are seen in molecular outflows induced on the cloud surrounding material. 
The forbidden lines, which form in low density regions, are direct indicators of the wind. Several important inferences can be obtained from the study of these lines. For instance, the line [OI] 6300 is present in all CTTS, even in those with weak emission, when the stellar spectrum is properly removed (Hartigan, Edwards, \& Ghandour 1995, HEG). As HEG show, the line profiles are composite, with a central component, whose origin is still under debate, and in stars with higher accretion, high velocity emission, generally blue-shifted, which at large scales merges into the jet. Disk occultation has been claimed as the explanation for the lack or a red-shifted counterpart to this emission (HEG). Interpretation of the forbidden line profiles have yielded estimates for the mass loss rates for CTTS, and the observed range is $10^{-9} M_{\odot} y r^{-1}-10^{-7} M_{\odot} y r^{-1}$ (HEG).

Forbidden lines indicate that the winds is powered by accretion. For instance, while [OI] 6300 is found in all CTTS, it is not found in any WTTS (HEG); forbidden line luminosities correlate with near infrared excesses (HEG), which in turn depend on accretion luminosities (Muzerolle et al. 2003c). Moreover, as shown in Figure 1, there is a very good correlation between mass loss rate and mass accretion rate. This correlation indicates that winds in CTTS do not have a stellar origin; the most likely origin region is the inner disk, where most of the accretion energy is released. However, the exact location and geometry of the region where the wind originates is still under discussion.

Essentially, two type of models have been presented for the region of origin of the wind, and I refer the reader to the appropriate references for the details of each. In here, I will describe their general characteristics and observational consequences, in particular for the emission of the inner disk regions. In all these models, the centrifugally-driven outflow follows field lines corotating with the launching point (Blandford \& Payne 1982). The condition is that a magnetic field is present, since the ionization fraction at the disk surface is enough for magnetic coupling (Gammie 1996). In one type of models, the stellar magnetic field is squeezed to the $\mathrm{X}$-point, where disk material is at corotation with the star. The wind is ejected from the $\mathrm{X}$-point, over a wide range of opening angles, and at high velocities, several hundreds of $\mathrm{km} \mathrm{s}^{-1}$ (Shu et al. 1994). In contrast, the disk wind models propose that the wind is ejected over a larger range of radii following field lines that go through the disk. It is ejected in a more restricted range of opening angles and a larger range of terminal velocities, which are proportional to the Keplerian velocities at the launching point (Pudritz \& Norman 1986; Pelletier \& Pudritz 1993; Königl 1989; Safier 1993). So, at small scales, each model has clearly different predictions for the emission.

\section{The blue-shifted absorption components}

Quantitative estimates of the wind properties from permitted emission lines in CTTS has proven difficult. Early attempts to determine mass loss rates from these lines assumed that the entire emission line was formed in the wind. However, since the bulk of the emission comes from the magnetospheric flow, as discussed, these attempts are no longer valid. The main wind indicators are the blue-shifted absorption components (BACs) superimposed on the emission lines. As shown in Figure 2, taken from Muzerolle et al. (2001), magnetospheric 


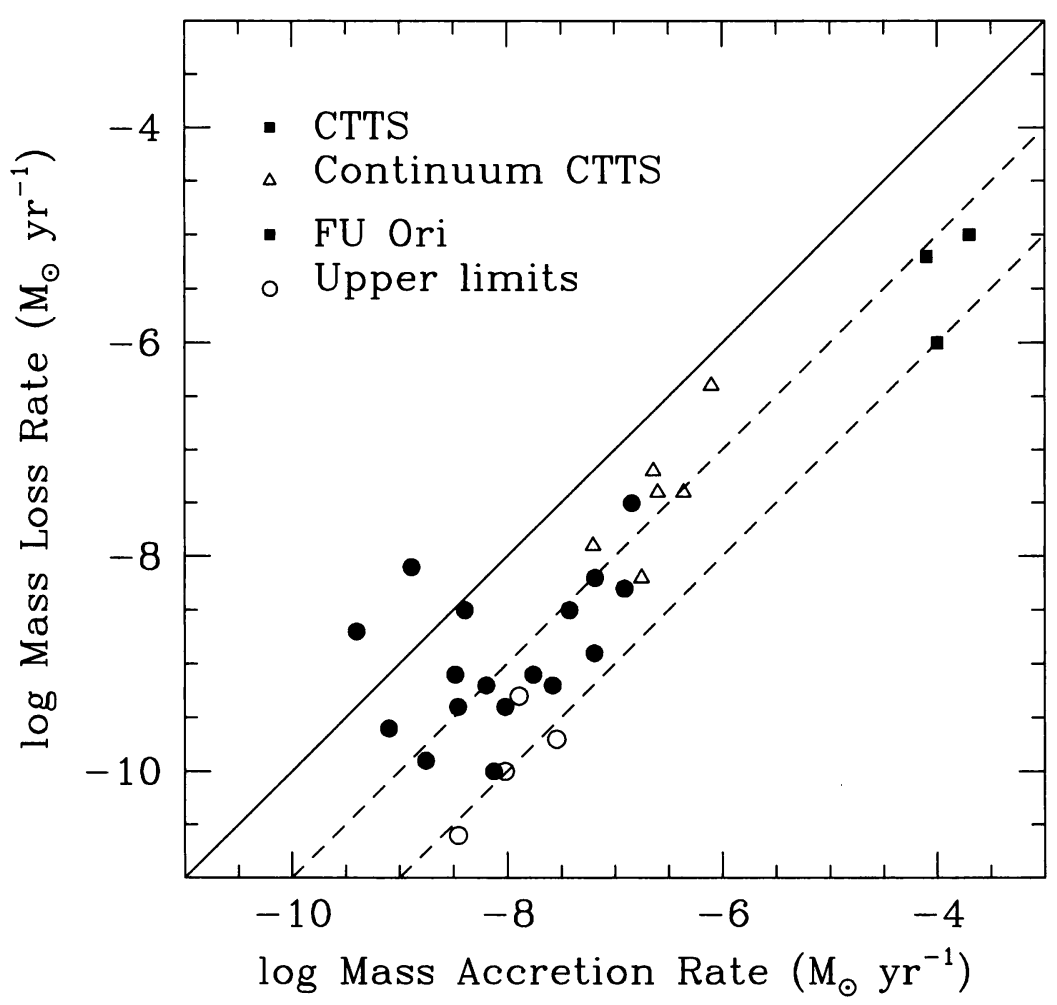

Figure 1. Mass loss rate vs. mass accretion rate for young stellar objects. Mass loss rates for CTTS are taken from Hartigan, Edwards, \& Ghandour (1995), while mass accretion rates are from Gullbring et al. (1998). Mass accretion rates for the continuum CTTS are from Gullbring et al. (2000). Mass accretion rates for the FU Ori objects are taken from Hartmann \& Kenyon (1996), while mass loss rates are from Calvet et al. (1993) and Hartmann \& Calvet (1995). The solid lines has slope 1. The two lower dashed lines correspond to slopes 0.1 and 0.01 . 
accretion can explain well the overall properties of the $\mathrm{H} \alpha$ and $\mathrm{Na}$ I D profiles. Only in the star with the highest mass accretion in the sample shown, DR Tau, the $\mathrm{H} \alpha$ profile is clearly $\mathrm{P}$ Cygni, and the magnetospheric component, showed as well, cannot account for the emission. Still, even in for this star, magnetospheric emission can explain the simultaneous $\mathrm{Na}$ I D profile. The emission of this lower opacity line is formed in the magnetosphere, and the wind appears only in a strong BAC.

The BAC is formed by material in the wind absorbing background emission mostly from the magnetosphere and accretion shock. This material must be relative close to the central object or along the line of sight. Thus, study of the $\mathrm{BAC}$ in lines of different excitation properties can give direct information on the geometry of the inner wind regions. This study is now underway (Alencar et al., in preparation).

Comparison of profiles of $\mathrm{H} \alpha$ and $\mathrm{Na} \mathrm{I} \mathrm{D} \mathrm{with} \mathrm{increasing} \mathrm{mass} \mathrm{accretion}$ rate shows that the strength of the BAC increases with $\dot{M}$ (Calvet 1998). In particular, the emission component is completely obliterated in the objects with highest mass accretion rate, the FU Ori objects, in which $\dot{M}$ has increased to $\sim 10^{-4} M_{\odot} y r^{-1}$ (Hartmann \& Kenyon 1996). At these values of $\dot{M}$, the magnetosphere would be crushed by the disk (see eq. [1]), and thus no emission is expected.

The FU Ori objects are clear examples of a disk wind. A definite trend is seen in the profiles of the atomic absorption lines formed in the inner disk atmospheres. Weak lines, formed on the disk photosphere, show the characteristic double line profile expected from a Keplerian disk; in contrast, strong lines, formed high in the wind region where expansion velocities are larger than rotational velocities, show single, blueshifted profiles (Calvet et al. 1993; Hartmann \& Calvet 1995).

Models of FU Ori winds have yielded mass loss rates. These are also shown in Figure 1. These measurements clearly continue the trend observed in the lower $\dot{M}$ CTTS, and suggest a common mechanism for the origin of the wind in all cases.

\section{High-resolution HST studies of the wind/outflow}

High spatial resolution studies give us clues about the geometry and physical conditions of the wind at small scales. In particular, cameras on board of HST yield resolutions of the order of $10 \mathrm{AU}$ at the closest star formation region, Taurus, allowing us to directly probe those regions.

The system of $\mathrm{T}$ Tau consists of two objects, the northern, visible star, and the embedded southern system, which is itself a binary (Duchêne, Ghez, \& McCabe 2002). The stars are surrounded by an infalling envelope (Calvet et al. 1994), traveling through which two powerful outflows have been identified. The NS outflow is marked by arcs of strong emission of infrared $\mathrm{H}_{2}$ lines, and is associated with the southern system. The EW outflow emerges from the northern component and is identified with a jet clearly seen in forbidden lines (Böhm \& Solf 1994; Solf \& Böhm 1999). At scales of a few thousand AU, two "rings" of ${ }^{13} \mathrm{CO}$ emission associated with this outflow have been identified by Momose et al. (1996). The small displacement between the center of the 


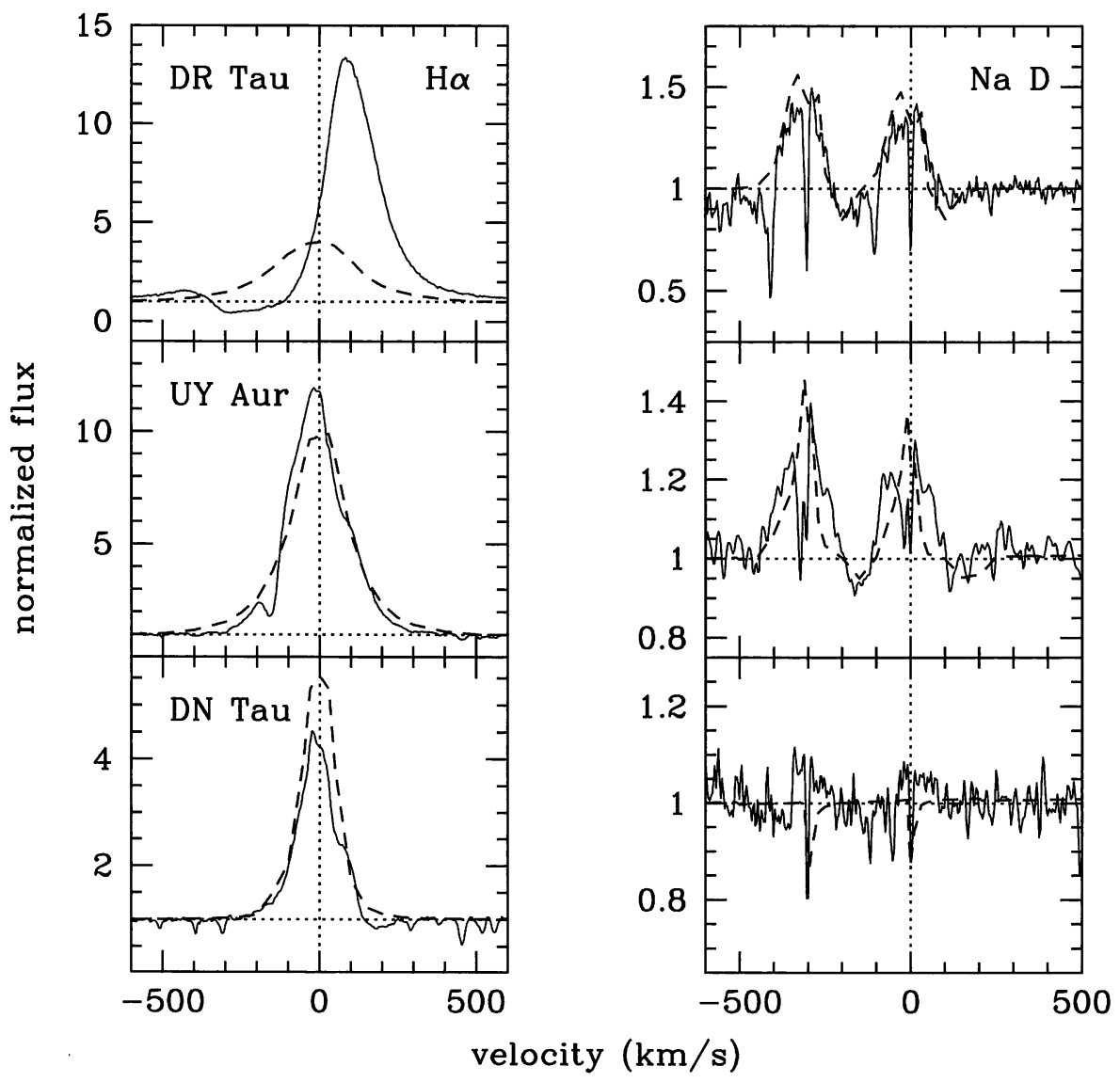

Figure 2. Line profiles of $\mathrm{H} \alpha$ (left column) and $\mathrm{Na}$ I D (right column) for the stars DR Tau, UY Aur, and DN Tau. The mass accretion rate ranges from $\sim 10^{-7} M_{\odot} y r^{-1}$ for DR Tau at the top to $\sim 10^{-9} M_{\odot} y r^{-1}$ for DN Tau at the bottom. Magnetospheric accretion model predictions are shown with dashed lines. Wind-type profiles are only present in the most powerful accretors and in the lines with highest opacity, namely $\mathrm{H} \alpha$. From Muzerolle et al. (2001). 
blueshifted and redshifted rings indicates a low inclination to the line of sight, $\sim 10^{\circ}$. These observations suggest that the EW outflow effectively opening a cavity in the envelope, and that we are looking down the aperture of this cavity. At smaller scales, < $100 \mathrm{AU}$, we can clearly see an arc-like edge illuminated by stellar light in an HST/WFPC image (Stapelfeldt et al. 1998).

HST/STIS far UV spectra of $\mathrm{T}$ Tau $\mathrm{N}$ shows extended emission, which consists of $\mathrm{H}_{2}$ lines which are produced by fluorescence with Lyman $\alpha$ radiation at $+98 \mathrm{~km} \mathrm{~s}^{-1}$ from line center (Saucedo et al. 2003, SCHR). The fact that the fluorescent $\mathrm{H}_{2}$ emission is extended has been known before (Brown 1981; Valenti et al. 2000). However, SCHR could spatially map the region where the emission arose, and they found that it follows the cavity in the envelope, see Figure 3. This emission was consistent with pumping by stellar Lyman $\alpha$. For the fluorescent mechanism to work, electrons must exist at excited levels of the ground state of the $\mathrm{H}_{2}$ molecule, which required temperatures of the order of $\sim 1000 \mathrm{~K}$. T Tau N, although more luminous than typical CTTS, is still not hot or luminous enough to heat envelope regions to this temperature at scales of some tens of AU. The most likely mechanism to achieve the required temperature is shock excitation. This shock could be created a the interface of a wide-angle wind emanating from the central object and the infalling envelope. Thus, these observations are clear evidence for wide-angle winds, and provide firm support to predictions of the X-wind theory that the optical jet is due to a density/temperature enhancement along the axis of the system, although the actual outflow covers a much wider area (Shang et al. 2002).

Other interesting observations in the present context are those of the forbidden line emission of the DG Tau jet with HST/STIS at scales down to $\sim 10$ AU by Bacciotti et al. $(2000 ; 2002)$. These observations map the emission at different velocity channels, and confirm that the high velocity blueshifted emission extends onto the optical jet. On the other hand, they find no high velocity redshifted emission near the source. This contradicts the predictions of the $\mathrm{X}$-wind model. Shang et al. (1998) have calculated the forbidden line emission expected for systems with different inclinations to the line of sight. For the inclination of $40^{\circ}$ of DG Tau (Eisloffel \& Mundt 1998), shifts to at least $+100 \mathrm{~km} \mathrm{~s}^{-1}$ should be detected near the source, which is not the case. This high positive velocity tail is a direct consequence of the large opening angle of the material as it leaves the X-point. Its absence seems to argue in favor of a more restricted range of angles for launching the wind, as expected from wind models.

\section{Summary and conclusions}

Winds/outflows in Classical $\mathrm{T}$ Tauri stars are powered by accretion. They are MHD winds originating in the inner parts of the disks, where most of the accretion energy is released. Comparison of the rates of accretion and loss of mass indicates that $\sim 0.1$ of the accreted matter is lost to the wind; the correlation holds over several orders of magnitude of accretion rate. Present observations indicate that the wind has a wide opening angle, which supports the $\mathrm{X}$-wind predictions that the optical jet results from a density enhancement towards the axis. On the other hand, this opening angle seems to more restricted than predicted by $\mathrm{X}$-wind calculations, favoring disk wind theories, similarly to the case 


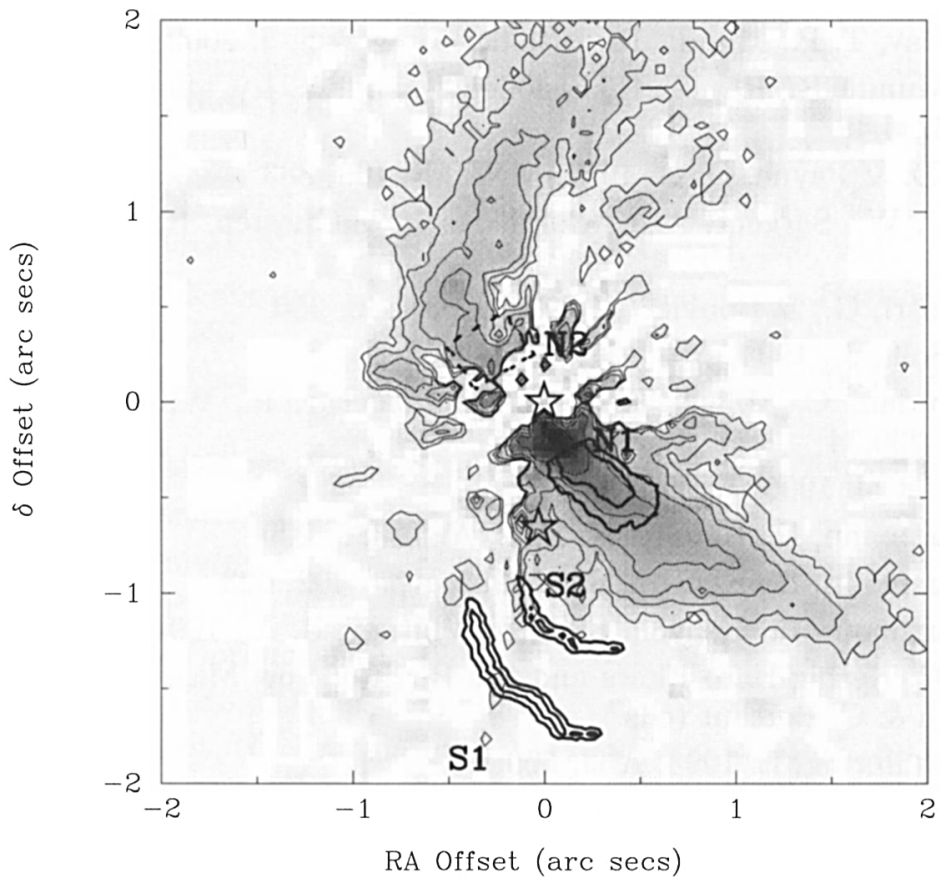

Figure 3. Spatial distribution of the $\mathrm{H}_{2}$ emission (thick solid lines). The fluorescent emission is superimposed on the magnitude isocontours of $0.55 \mu \mathrm{m}$ emission from Stapelfeldt et al. (1998) (gray scale/thin lines). The $\mathrm{H}_{2}$ emission traces the edge of the envelope cavity delineated by the $0.55 \mu \mathrm{m}$ emission. The two arcs at the south are probably associated with the NS outflow (from Saucedo et al. 2003). 
of FU Ori objects. To generalize and get a firmer knowledge of the structure of the region where the wind originates, observations of more objects are required, as well as detailed observational predictions from all theoretical models.

Acknowledgments. Research presented here was supported by NASA through grants GO-08317 and GO-09081 from the Space Telescope Science Institute, and by NASA Origins of Solar Systems grant NAG5-9670.

\section{References}

Bacciotti, F., Ray, T. P., Mundt, R., Eislöffel, J., \& Solf, J. 2002, ApJ, 576, 222 Bacciotti, F., Mundt, R., Ray, T. P., Eislöffel, J., Solf, J., \& Camezind, M. 2000, ApJ, 537, L49

Blandford, R. D. \& Payne, D. G. 1982, MNRAS, 199, 883

Beckwith, S. V. W., Sargent, A. I., Chini, R. S., \& Guesten, R. 1990, AJ, 99, 924

Bertout, C., Basri, G., \& Bouvier, J. 1988, ApJ, 330, 350

Böhm, K., \& Solf., J., 1994, ApJ 430, 277-290

Brown, A., Jordan, C, Millar, T. J., \& Gondhalekar, P., Wilson, R., 1981, Nature, 290,34

Burrows, C. J. et al. 1996, ApJ, 473, 437

Calvet, N., Hartmann, L., Hewett, R. 1992, ApJ, 386, 229

Calvet, N., Hartmann, L., Kenyon, S. J. 1993, ApJ, 402, 623

Calvet, N., Hartmann, L., Kenyon, S. J., \& Whitney, B. A., 1994, ApJ 434,330

Calvet, N. 1997, Herbig-Haro Flows and the Birth of Low Mass Stars, 417, B. Reipurth \& C. Bertout (eds.)

Calvet, N. \& Gullbring, E. 1998, ApJ, 509, 802

Costa, V. M., Lago, M. T. V. T., Norci, L., \& Meurs, E. J. A. 2000, A\&A, 354, 621

Duchêne, G., Ghez, A., \& McCabe, C., 2002, ApJ 568, 771

Edwards, S., Hartigan, P., Ghandour, L. \& Andrulis, C. 1994, AJ, 108, 1056

Eislöffel, J., \& Mundt, R., 1998, AJ 115, 1554

Gammie, C. F. 1996, ApJ, 457, 355

Ghosh, P. \& Lamb, F. K. 1979, ApJ, 234, 296

Giampapa, M. S., Calvet, N., Imhoff, C. L., \& Kuhi, L. V. 1981, ApJ, 251, 113

Gullbring, E., Hartmann, L., Briceño, C., \& Calvet, N. 1998, ApJ, 492, 323

Gullbring, E., Calvet, N., Muzerolle, J., Hartmann, L., 2000, ApJ 544, 927

Hartmann, L. 1998, Accretion Processes in Star Formation, Cambridge University Press

Hartmann, L., Edwards, S., Avrett, E.: 1982, ApJ, 261, 279

Hartmann, L., Calvet, N., Avrett, E. H., Loeser, R. 1990, ApJ, 349, 168

Hartmann, L., Hewett, R., \& Calvet, N. 1994, ApJ, 426, 669

Hartmann, L., Calvet, N. 1995, AJ., 109, 1846 
Hartmann, L., Kenyon, S. J. 1996, ARAA, 34, 207

Hartigan, P., Edwards, S., \& Ghandour, L., 1995, ApJ, 452, 736

Kenyon, S. J. \& Hartmann, L. 1987, ApJ, 323, 714

Königl, A. 1989, ApJ, 342, 208

Königl, A. 1991, ApJ, 370, L39

Lynden-Bell, D. \& Pringle, J. E. 1974, MNRAS, 168, 603

Johns-Krull, C. M., Valenti, J. A., Hatzes, A. P., \& Kanaan, A. 1999a, ApJ, 510, L41

Johns-Krull, C. M., Valenti, J. A., \& Koresko, C. 1999b, ApJ, 516, 900

Kuhi, L. V. 1964, ApJ, 140, 1409

Momose, M., Nagayoshi, O., Kawabe, R., Masahiko, H., \& Nakano, T., 1996, ApJ, 470, 1001-1014

Muzerolle, J., Calvet, N., \& Hartmann, L. 1998, ApJ, 492, 743

Muzerolle, J., Hartmann, L., \& Calvet, N. 1998, AJ, 116, 455

Muzerolle, J., Calvet, N., \& Hartmann, L. 2001, ApJ, 550, 944

Muzerolle, J., Hillenbrand, L., Calvet, N., Briceño, C., \& Hartmann, L. 2003a, ApJ, 592, 266

Muzerolle, J., D'Alessio, P., Calvet, N., \& Hartmann, L. 2003b, ApJ, (submitted)

Muzerolle, J., Calvet, N., Hartmann, L., \& D'Alessio, P. 2003c, ApJ, 597, L149

Natta, A., Giovanardi, C. 1990, ApJ, 356, 646

Natta, A., Giovanardi, C., Palla, F. 1988, ApJ, 332, 921

Pelletier, G., \& Pudritz, R.E. 1992, ApJ, 394, 117

Pudritz, R.E., \& Norman, C.A. 1986, ApJ, 301, 571

Rucinski, S. M. 1985, AJ, 90, 2321

Saucedo, J., Calvet, N., Hartmann, L., \& Raymond, J. 2003, ApJ, 591, 275

Safier, P. 1993, ApJ, 408, 115

Shang, H., Glassgold, A., Shu, F., \& Lizano, S., 2002, ApJ 564, 853

Solf, J., \& Böhm, K. H., 1999, ApJ 523,709

Stapelfeldt, K. et al. 1998, ApJ 508, 74

Shu, F., Najita, J., Ostriker, E., \& Wilkin, F. 1994, ApJ, 429, 781

Valenti, J., Johns-Krull, C.M., \& Linsky, J., 2000, ApJS, 129,399

Walker, M. F. 1972, ApJ, 175, 89

Wolf, B., Appenzeller, I., \& Bertout, C. 1977, A\&A, 58, 163 

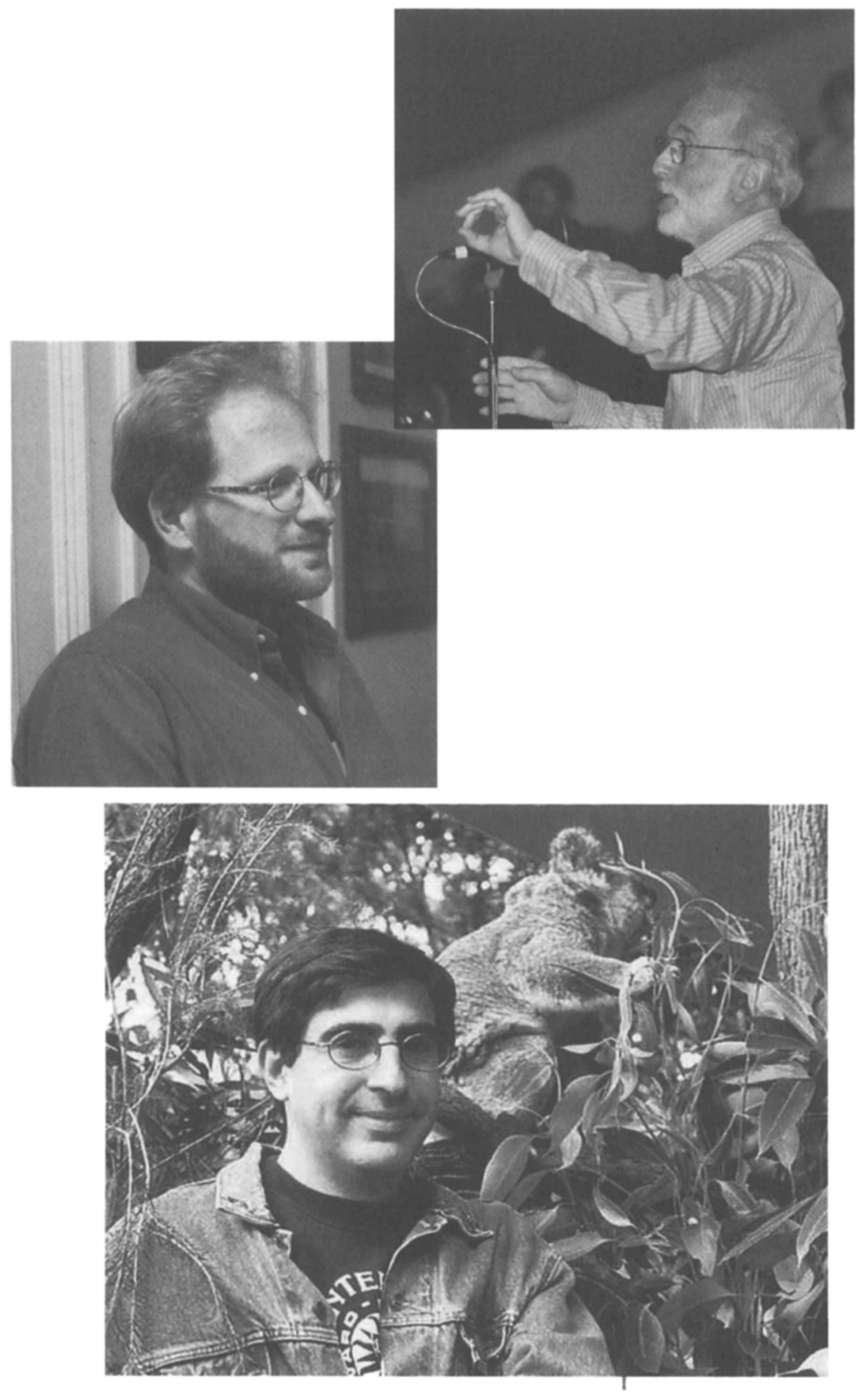